\title{
Riesgos Debido a la Radiactividad Natural de Pizarras de Construcción
}

\author{
Conrado Miró ${ }^{1}$, José M. Ávila ${ }^{2}$, Máximo García ${ }^{3}$ y José Pastor-Villegas ${ }^{4}$ \\ (1) Universidad de Extremadura, Facultad de Veterinaria, Departamento de Física Aplicada, \\ Avda. de la Universidad s/n, 10071 Cáceres-España (e-mail:cmiro@unex.es) \\ (2) INTROMAC, Departamento de I+D, Avda. de la Universidad s/n, 10071 Cáceres-España \\ (3) ICMC, Departamento de Control de Calidad, c/ Pamplona s/n, 06800 Mérida-España \\ (4) Universidad de Extremadura, Facultad de Formación del Profesorado, Departamento de Química \\ Orgánica e Inorgánica, Avda. de la Universidad s/n, 10071 Cáceres-España
}

\section{Resumen}

El objetivo de este trabajo es medir la radiactividad natural en pizarras utilizadas como material de construcción. Se analizaron pizarras fabricadas en la región de Extremadura-España como caso de estudio. Las concentraciones se determinaron por espectrometría gamma utilizando detectores coaxiales de HPGe. Se determinaron las actividades equivalente de radio y varios índices de riesgo. Así mismo se compararon los resultados con los obtenidos en la bibliografía en otros países del mundo. Los resultados muestran que los valores medios de concentración debido al ${ }^{40} \mathrm{~K},{ }^{226} \mathrm{Ra}$ y ${ }^{232} \mathrm{Th}$ fueron 840,29 y $75 \mathrm{~Bq} / \mathrm{kg}$, respectivamente. La actividad equivalente de radio fue inferior al valor de $370 \mathrm{~Bq} / \mathrm{kg}$ establecido como norma, y los otros índices de riesgo también fueron inferiores a sus límites.

Palabras clave: radiactividad natural, pizarra de construcción, índice de riesgo, espectroscopia

\section{Natural Radioactivity Hazards produced by Construction Slates}

\begin{abstract}
The aim of this work is to measure the natural radioactivity in slates used as building material. Slates manufactured in the region of Extremadura-Spain were used as a study case. The concentrations were determined by gamma spectrometry using an HPGe coaxial detector. Radium equivalent activities $\left(\mathrm{Ra}_{\mathrm{eq}}\right)$ and various hazard indices were also calculated. Results were also compared with the data available in the literature for other countries of the world. The results show that the average values of the concentrations due to ${ }^{40} \mathrm{~K},{ }^{226} \mathrm{Ra}$ and ${ }^{232} \mathrm{Th}$ were found 840,29 and $75 \mathrm{~Bq} / \mathrm{kg}$, respectively. The radium equivalent activity was less than the accepted standard criterion value of $370 \mathrm{~Bq} / \mathrm{kg}$ and the values of other hazard indices were also below their limit values.
\end{abstract}

Keywords: natural radioactivity, construction slate, hazard index, spectroscopy 


\section{INTRODUCCIÓN}

La radiactividad natural es una parte de nuestro mundo (Eisenbud, 1987). Nuestro planeta Tierra contiene diferentes tipos de especies radiactiva de origen natural (Kathren, 1998). Ello se debe a las radiaciones emitidas por las desintegraciones naturales los miembros de las cadenas de desintegración del uranio y torio y también por el ${ }^{40} \mathrm{~K}$. El ${ }^{238} \mathrm{U}$ y el ${ }^{232} \mathrm{Th}$ están presentes en la corteza terrestre en partes por millón (Evans, 1969). El potasio natural está también presente en dicha corteza y un $0.0118 \%$ del total de este potasio es ${ }^{40} \mathrm{~K}$, el cual es radiactivo. Así pues, los estudios sobre la radiactividad natural tienen una enorme importancia debido a que es la principal fuente de exposición de la radiación al ser humano.

Además, los materiales de construcción son una de las fuentes que causan una exposición directa a la radiación. El conocimiento de los parámetros radiológicos básicos y los contenidos radiactivos en los materiales de construcción tiene importancia ya que permite calcular la exposición de la población a la radiación a partir de las fuentes naturales (Ali et al., 1996; Lee et al., 2004, Tufail et al., 2007). El cemento, ladrillo, arcilla, y rocas graníticas pueden producir tasas de dosis significativas, de 20 a 190 $\mathrm{nGy/h}$, dependiendo del tipo y del lugar de la construcción, pudiendo contribuir hasta un cuarto de la dosis total anual que el público recibe de las fuentes de radiación (UNSCEAR, 2000). Además de los materiales aplicados a las estructuras de una construcción, materiales usados para la decoración de habitaciones también pueden ser considerados como posibles fuentes de radiactividad en interiores, como por ejemplo, materiales cerámicos. Estos materiales pueden contener sales de uranio con objeto de darles colores.

Así pues, el conocimiento de la concentración de estos radionúclidos en los materiales juega un papel importante en las medidas de protección contra la radiación. Por ello, numerosos países han desarrollado estudios para compilar datos sobre su peligrosidad radiológica (ver por ejemplo, Ahmed, 2005; Amrani y Tahtat, 2001; Beretka y Mathew, 1985; Farai y Ademola, 2005; Hewamanna et al., 2001; Lee et al., 2004; Khan, et al.,2002).

Entre los materiales usados en edificios está la piedra natural la cual puede producir tasas significativas de dosis externa (Asghar et al., 2008). El objetivo del presente trabajo se centra en un tipo de piedra natural, la pizarra, utilizada como material de construcción. Concretamente se trata de determinar el impacto radiológico de pizarras extraídas en canteras de la región de Extremadura (España). Para ello, en primer lugar, se ha determinado el contenido en ${ }^{226} \mathrm{Ra},{ }^{232} \mathrm{Th}$ y ${ }^{40} \mathrm{~K}$. Los datos resultantes se han comparado con los encontrados por otros países del mundo. Seguidamente, se han determinado diversos índices de riesgo con objeto de valorar la peligrosidad de la radiación procedente de este tipo de roca y se relacionan con los límites de seguridad recomendados por la ICRP (1977).

\section{PROCEDIMIENTO EXPERIMENTAL}

\section{Muestras}

Los materiales pizarrosos son muy abundantes dentro de la región de Extremadura, situada al suroeste de España. Se presentan tanto en el dominio Centro -lbérico como en el de Ossa Morena, y abarcan materiales comprendidos desde el Precámbrico al Carbonífero. Presentan una gran variedad litológica de unos pisos a otros, así como dentro del mismo nivel. Los términos más frecuentes son pizarras arcillosas y areniscas, intercaladas generalmente en series esquistosas más o menos potentes. En la actualidad únicamente los niveles pizarrosos del Silúrico Superior Devónico Medio del área de Alburquerque - La Roca de la Sierra presentan una explotación activa y continuada. Este tipo de explotaciones presenta como principal problema, la gran cantidad de escombreras que genera. Se estima que de todo lo extraído de cualquier cantera de pizarras, solamente es vendible una cantidad aproximada comprendida entre el 2 y el $10 \%$. Esto significa que el resto pasa a formar parte de las escombreras.

La exploración de posibles aprovechamientos de estos residuos pasa por otras aplicaciones. Dependiendo del tamaño que presenten los fragmentos de pizarra se puede presumir su posible empleo, así los fragmentos de tamaño arena o grava se pueden destinar para la fabricación de 
piedra artificial, mezclas asfálticas y en la elaboración de cierto tipo de prefabricados. Los tamaños más finos se pueden destinar a la fabricación de compuestos bituminosos, plásticos, cauchos, pinturas, fertilizantes, insecticidas, estabilizantes de $\mathrm{pH}$ etc, siempre bajo un estricto control de la granulometría y composición.

Los niveles de concentración radiactiva o actividad específica se determinaron a partir de 5 muestras de pizarra recolectadas en el área de transición entre las zonas Centro - Ibérica y Ossa -Morena, dentro del denominado sinclinorio de Sao Mamede- La Codosera. Se trata de pizarras negras político - grafitosas muy compactas, que constituyen una litofacies restringida a determinadas zonas, del flanco sur del sinclinorio. Las principales características generales de este material son: color negro, contenido en carbonatos menor del 0,68\%, coeficiente de absorción del $0.13 \%$ y resistencia a la flexión de 54.26 MPa (INTROMAC, 2009). Más información acerca de la geología de los suelos de la región puede consultarse en los mapas geológicos de Extremadura, (ver por ejemplo SIGEO, 2009).

Una vez recolectadas, las muestras se trituraron en un molino de bolas, a continuación, fueron tamizadas para conseguir un tamaño de partícula menor de $2 \mathrm{~mm}$. Las muestras fueron homogeneizadas y secadas en estufa durante 48 horas a $95^{\circ} \mathrm{C}$ para eliminar la humedad. Por último, fueron empaquetadas en viales cilíndricos de plástico de $90 \mathrm{~mm}$ de diámetro y $31 \mathrm{~mm}$ de altura, anotando el peso seco de dicha muestra. Los recipientes debidamente sellados se almacenaron durante 40 días antes de las medidas, para conseguir que el ${ }^{226} \mathrm{Ra}$ alcanzase el equilibrio secular radiactivo con sus productos de desintegración de vida corta, como el ${ }^{214} \mathrm{~Pb}$ y el ${ }^{214} \mathrm{Bi}$.

\section{Medida de radiactividad}

La concentración de radiactividad natural $\left({ }^{226} \mathrm{Ra},{ }^{232} \mathrm{Th}\right.$ y $\left.{ }^{40} \mathrm{~K}\right)$ se determinó utilizando un sistema de espectrometría gamma compuesto por un detector coaxial de Germanio Hiperpuro (Ge HP) tipo-n, acoplado a un analizador multicanal de 4096 canales. El detector tiene una eficiencia del $25.6 \%$, una resolución (FWHM) de $1.85 \mathrm{keV}$ y una relación pico-Compton de $57: 1$ para la emisión del ${ }^{60} \mathrm{Co}$ a $1332.5 \mathrm{kev}$. El espectro gamma se analizó usando el programa de ordenador GAMMAVISION. EI sistema de detección se había calibrado previamente en función de la energía de emisión, utilizando el coctel QCY.48 de Amersham en una matriz de calibración de suelo de similar densidad a la de la pizarra en polvo.

El cálculo de las actividades específicas se obtuvo a partir del promedio de los fotopicos a las diferentes energías. Para el ${ }^{226} \mathrm{Ra}$ se utilizaron las líneas de emisión gamma del ${ }^{214} \mathrm{Bi}(609.3$ y 1764.5 $\mathrm{keV})$ y del ${ }^{214} \mathrm{~Pb}(351.9 \mathrm{keV})$. Para la determinación del ${ }^{232} \mathrm{Th}$, las líneas de emisión fueron las del ${ }^{228} \mathrm{Ac}(911 \mathrm{keV})$ y ${ }^{208} \mathrm{TI}(583.01 \mathrm{keV})$. El ${ }^{40} \mathrm{~K}$ se determinó directamente a partir de su fotopico gamma a $1460.7 \mathrm{keV}$. Las medidas de las muestras se realizaron durante $24 \mathrm{~h}$, y previamente se había efectuado una medida del fondo ambiental durante $48 \mathrm{~h}$. La actividad específica $A$, medida en Bq/kg, se determinó a partir de la siguiente relación:

$A=R_{n} /(\varepsilon P M)$

donde $R_{n}$ es la tasa de conteo neta (conteo de la muestra menos el conteo del fondo radiactivo ambiental) (cuentas por segundo), $\varepsilon$ es la eficiencia del detector para cada línea de emisión, $P$ es la probabilidad de desintegración de cada fotón gamma y $M$ es la masa de la muestra medida (kg).

\section{RESULTADOS Y DISCUSIÓN}

\section{Actividad específica}

Los valores medios, y sus desviaciones estándar (SD), de la actividad específica obtenidos en este trabajo fueron $840 \pm 40$ (SD), $29 \pm 4$ (SD) y $75 \pm 10$ (SD) Bq/kg, para el ${ }^{40} \mathrm{~K},{ }^{226} \mathrm{Ra}$ y ${ }^{232} \mathrm{Th}$, respectivamente. De los valores de las desviaciones estándar se deduce que la dispersión de los valores de concentración entre las distintas muestras es relativamente pequeña. Por otro lado, el cociente entre actividades ${ }^{232} \mathrm{Th} /{ }^{226} \mathrm{Ra}$ fue mayor que la unidad en todas las muestras analizadas, lo 
cual está en concordancia con los datos de fondo global sobre concentraciones radiactivas en la corteza de la Tierra (Schmus, 1995).

Con objeto de comparar dichos resultados con los obtenidos por otros autores, se ha realizado una revisión bibliográfica sobre contenidos radiactivos en distintos países del mundo. Estos trabajos muestran las concentraciones medias en diferentes materiales de construcción, entre los que se incluyen el ladrillo, el cemento, yeso, materiales cerámicos y también, lógicamente, las piedras naturales (granitos, mármoles, pizarras, etc.). Dichos resultados se muestran en la Tabla 1. También en la penúltima fila de la tabla se indican los valores encontrados en este trabajo y en la última fila, los valores medios juntos con los rangos de variación promedios que estima la UNSCEAR (2000) a nivel mundial.

Como se deduce de dicha tabla, hay en general una gran dispersión de datos en los distintos países. Los resultados del presente estudio, para los tres radionúclidos de interés entran dentro del rango de los valores reportados en la bibliografía. Aunque para el ${ }^{40} \mathrm{~K}$ y el ${ }^{232} \mathrm{Th}$ la pizarra extremeña presenta un valor aproximadamente el doble del valor medio mundial, superando la cota superior de valores medios en el caso del torio, y para el ${ }^{226} \mathrm{Ra}$ es ligeramente inferior al estimado para todo el mundo.

Tabla 1: Comparación de las actividades específicas: valores obtenidos en distintos países, y valores promedios mundiales junto con sus rangos de variación promedios (entre paréntesis).

\begin{tabular}{|c|c|c|c|c|}
\hline \multicolumn{5}{|c|}{ Actividad específica media de los radionúclidos (Bq/kg) } \\
\hline País & ${ }^{40} \mathrm{~K}$ & ${ }^{226} \mathrm{Ra}$ & ${ }^{232} \mathrm{Th}$ & Referencia \\
\hline Alemania & 670 & 59 & 67 & Eisenbud (1987) \\
\hline Argelia & 310 & 23 & 18 & Amrani y Tahtat (2001) \\
\hline Austria & 635 & 38 & 45 & Sorantin y Steger (1984) \\
\hline Australia & 681 & 41 & 89 & Beretka y Mathew (1985) \\
\hline Bangladesh & 234 & 109 & 58 & Mollah et al. (1986) \\
\hline Bélgica & --- & 40 & 33 & Proffijin et al. (1984) \\
\hline Brasil & 747 & 52 & 65 & Malanca et al. (1993) \\
\hline Canadá & 800 & 37 & 49 & Zikovsky y Kennedy (1992) \\
\hline China & 714 & 59 & 50 & Xinwei (2005) \\
\hline Egipto & 852 & 187 & 118 & Ahmed (2005) \\
\hline Finlandia & 986 & 80 & 62 & Mustonen (1984) \\
\hline Grecia & 670 & 49 & 24 & Papastefanou et al. (1984) \\
\hline Holanda & 560 & 39 & 41 & Ackers et al. (1985) \\
\hline India & 47 & 13 & 74 & Kumar et al. (2003) \\
\hline Italia & 694 & 47 & 50 & Sciocchetti et al. (1984) \\
\hline Irlanda & 353 & 32 & 18 & Lee et al. (2004) \\
\hline Malasia & 684 & 233 & 229 & Chong y Ahmad (1982) \\
\hline Méjico & --- & 16 & 23 & Espinosa et al. (1986) \\
\hline Nigeria & 253 & 16 & 36 & Farai y Ademola (2005) \\
\hline Pakistán & 693 & 45 & 62 & Tufail et al. (2007) \\
\hline Polonia & 573 & 35 & 50 & Niewiadomski y Wasiolek (1985) \\
\hline Sri Lanka & 583 & 72 & 35 & Hewamanna et al. (2001) \\
\hline España & 840 & 29 & 75 & Este trabajo \\
\hline Mundo & $\begin{array}{l}400 \\
(140-850)\end{array}$ & $\begin{array}{l}35 \\
(17-60)\end{array}$ & $\begin{array}{l}30 \\
(11-64)\end{array}$ & UNSCEAR (2000) \\
\hline
\end{tabular}

Los resultados de este trabajo pueden compararse también, por ejemplo, con el contenido radiactivo de los suelos, ya que éstos pueden contener importantes concentraciones radiactivas de origen natural. En un trabajo anterior realizado en el laboratorio, se encontró que el valor medio de los suelos, a nivel superficial (hasta $5 \mathrm{~cm}$ de profundidad), en Extremadura (Baeza et al., 1994) fue de $653,38.3$ y $41.0 \mathrm{~Bq} / \mathrm{kg}$ para el ${ }^{40} \mathrm{~K},{ }^{226} \mathrm{Ra} \mathrm{y}{ }^{232} \mathrm{Th}$, respectivamente. Por lo que el cociente entre las concentraciones en pizarra y las de suelos son $1.29,0.67$ y 1.8 para el ${ }^{40} \mathrm{~K},{ }^{226} \mathrm{Ra}$ y ${ }^{232} \mathrm{Th}$, 
respectivamente. Es decir, para la piedra natural estudiada son algo superiores en ${ }^{40} \mathrm{~K}$ y ${ }^{232} \mathrm{Th}$ e inferiores en ${ }^{226} \mathrm{Ra}$ a la de los suelos.

\section{Índices radiológicos de riesgo}

El fin último de la medida de las concentraciones radiactivas es determinar la peligrosidad de la radiación procedente de estos materiales. Dicha peligrosidad se mide mediante la determinación de los denominados "índices radiológicos de riesgo". Los índices más utilizados son la "actividad equivalente de radio", el "índice de riesgo externo", el "índice de riesgo interno" y el "índice de concentración de actividad gamma".

La actividad equivalente de radio, $R a_{e q}$, se utiliza para comparar la actividad específica de los materiales que contienen distintas cantidades de ${ }^{40} \mathrm{~K},{ }^{226} \mathrm{Ra}$ y ${ }^{232} \mathrm{Th}$. $R a_{\text {eq }}$ se ha calculado, en $\mathrm{Bq} / \mathrm{kg}$, a partir de la siguiente relación (Beretka y Mathew, 1985):

$R a_{e q}=\left(A_{T h} \times 1.43\right)+A_{R a}+\left(A_{K} \times 0.077\right)$

siendo $A_{\mathrm{Th}}, A_{\mathrm{Ra}}$ y $A_{\mathrm{K}}$ las actividades específicas del ${ }^{232} \mathrm{Th},{ }^{226} \mathrm{Ra}$ y ${ }^{40} \mathrm{~K}$, respectivamente, medidas en $\mathrm{Bq} / \mathrm{kg}$.

Se asume una actividad equivalente de radio de $370 \mathrm{~Bq} / \mathrm{kg}$ en materiales de construcción producirá una exposición de alrededor de 1.5 mGy/año en la población (Krieger, 1981), valor considerado como límite máximo por la ICRP (1977).

Aplicando la ecuación (2) se obtiene para las pizarras estudiadas una actividad equivalente de radio de $201 \mathrm{~Bq} / \mathrm{kg}$. Los niveles máximos recomendados de radio equivalente para los materiales de construcción que vayan a ser utilizados en hogares son $370 \mathrm{~Bq} / \mathrm{kg}$ y para industrias es $740 \mathrm{~Bq} / \mathrm{kg}$. (Oresegun y Babalola, 1988). Por tanto, el valor obtenido para las pizarras se sitúa inferior al límite máximo recomendado para viviendas. Por otro lado, se encuentra dentro del rango de valores medios mundiales estimados por la UNSCEAR (2000) que resulta ser (43.5-216.8) Bq/kg, aunque estaría próximo al valor máximo de dicho rango.

El índice de riesgo externo, $H_{e x}$, es otro criterio para determinar la peligrosidad de un material de construcción. Se define (Beretka y Mathew, 1985) según la expresión:

$H_{\text {ex }}=\frac{{ }^{A_{T h}}}{259}+\frac{{ }^{A_{R a}}}{370}+\frac{{ }^{A_{K}}}{4810}$

El valor de este índice debe ser menor o igual que la unidad para que la dosis anual externa recibida por el índividuo como consecuencia de la radiación gamma sea menor o igual que $1.5 \mathrm{mGy}$. El valor obtenido para las pizarras utilizadas en este trabajo fue de 0.54 y por tanto menor que la unidad

Además de la irradiación externa del organismo, el radón y sus productos de desintegración de vida corta también son peligrosos para la salud ya que al estar en forma gaseosa son inhalados por las personas, sobre todo cuando están en el interior de habitaciones. El "índice de riesgo interno", $H_{\text {in }}$, es un criterio para estimar la peligrosidad de la radiación en ambientes interiores. Dicho índice se define (Beretka y Mathew, 1985) como:

$H_{\text {in }}=\frac{A_{T h}}{259}+\frac{A_{R a}}{185}+\frac{A_{K}}{4810}$

Para el uso seguro de materiales de construcción utilizados en interiores de viviendas Krieger (1981) propone que $H_{\text {in }} \leq 1$. 
Para las pizarras estudiadas dicho índice resulta ser igual a 0.62 , por tanto menor que 1 , además estaría dentro del rango de valores medios mundiales (UNSCEAR, 2000) que resulta ser (0.16-0.74), aunque próximo a su extremo superior.

Otro de los índices de riesgo de la radiación es el denominado "índice de concentración de actividad gamma", $I_{V}$, definido por la Comisión Europea (EC, 1999) y dado por la expresión:

$$
I_{\gamma}=\frac{A_{T h}}{200}+\frac{A_{R a}}{300}+\frac{A_{K}}{3000}
$$

El índice $I_{V}$ está correlacionado con la tasa de dosis anual debida al exceso de radiación gamma externa causada por materiales de superficie. Valores del índice $I_{Y} \leq 2$ corresponde a un criterio de tasa de dosis de $0.3 \mathrm{mSv} / a n ̃ o$, mientras que $2<I_{V} \leq 6$ corresponde a un criterio de $1 \mathrm{mSv} /$ año (EC, 1999). Así este índice de concentración de actividad debería ser utilizado sólo como herramienta para distinguir aquellos materiales que puedan ser utilizados en construcción de los que no se recomienda su utilización, de tal manera que materiales con $I_{V}>6$ deberían evitarse ya que pueden dar lugar a tasas de dosis superiores a $1 \mathrm{mSv} / \mathrm{año}$ (EC, 1999).

Para las pizarras estudiadas en el presente trabajo dicho índice resulta ser igual a 0.75 , por tanto este material puede utilizarse sin ninguna restricción desde el punto de vista radiológico. Además estaría dentro del rango de valores medios mundiales (UNSCEAR, 2000) que resulta ser (0.16-0.84), aunque es mayor que el valor medio mundial de 0.40 .

\section{CONCLUSIONES}

En este trabajo se han determinado las actividades específicas de ${ }^{226} \mathrm{Ra},{ }^{232} \mathrm{Th}$ y ${ }^{40} \mathrm{~K}$ en pizarras extraídas de la región de Extremadura (España), mediante el uso de técnicas de espectrometría gamma con un detector de Germanio Hiperpuro. Las concentraciones de estos radionúclidos entran dentro del rango de los valores reportados en la bibliografía, si bien, para el ${ }^{40} \mathrm{~K}$ y el ${ }^{232} \mathrm{Th}$ la pizarra extremeña presenta un valor aproximadamente el doble del valor medio mundial, superando la cota superior de valores medios en el caso del torio, y para el ${ }^{226} \mathrm{Ra}$ es ligeramente inferior al estimado para todo el mundo. Los valores de los índices de riesgo derivados de las medidas de la actividad están por debajo de los valores límites permitidos. Así el valor de $R a_{e q}$ fue de unos $200 \mathrm{~Bq} / \mathrm{kg}$ por tanto, inferior a $370 \mathrm{~Bq} / \mathrm{kg}$ y, además, $H_{e x}<1$. Ello implica que la dosis por radiación gamma recibida externamente por un ocupante de una habitación conteniendo estos materiales es menor que 1.5 mGy/año. Por otro lado, al ser el índice de riesgo interno, $H_{i n}$, menor que 1 , tampoco existirán problemas significativos debido a la inhalación del gas radón. Por último, el índice de concentración de actividad es de 0.75 , muy inferior al límite máximo estimado como peligroso para los materiales usados en construcción. En comparación con otros países del mundo, los resultados encontrados en este trabajo se sitúan dentro de los rangos de valores medios pero próximos al extremo superior.

\section{AGRADECIMIENTOS}

Al financiamiento otorgado de la Junta de Extremadura-fondos FEDER, para el proyecto "Radiactividad Natural en los Materiales de Construcción" (PRI06A258)

\section{NOMENCLATURA}

A Actividad específica o concentración

$A_{\text {Th }} \quad$ Actividad específica del ${ }^{232} \mathrm{Th}$

$A_{\mathrm{Ra}} \quad$ Actividad específica del ${ }^{226} \mathrm{Ra}$

$A_{\mathrm{K}} \quad$ Actividad específica del ${ }^{40} \mathrm{~K}$

$H_{e x} \quad$ Índice de riesgo externo

$H_{\text {in }} \quad$ Índice de riesgo interno

$I_{Y} \quad$ Índice de concentración de actividad gamma

$M \quad$ Masa de la muestra medida

$P \quad$ Probabilidad de desintegración de cada fotón gamma 
$R_{n} \quad$ Tasa de contaje neta o contaje de la muestra menos el del fondo radiactivo

$R a_{e q} \quad$ Actividad equivalente de radio

$\varepsilon \quad$ Eficiencia del detector para cada línea de emisión

\section{REFERENCIAS}

Ackers, J.G., J.F Den-Boer, P. De-Jong y R.A Wolschrijn; Radioactivity and exhalation rates of building materials in the Netherlands, Sci. Total Environ.: 45, 151-156 (1985).

Ahmed, N.K.; Measurement of natural radioactivity in building materials in Qena city, Upper Egypt, J. Environ. Radioactivity: 83, 91-99 (2005).

Ali, S. y otros cuatro autores; Gamma-ray activity and dose rate of brick samples from some areas of North West Frontier Province (NWFP), Pakistan. Sci. Total Environ.: 187, 247-252 (1996).

Amrani, D. y M. Tahtat; Natural radioactivity in Algerian building materials, Appl. Radiat. Isotopes: 54, 687-689 (2001).

Asghar, M. y otros cuatro autores; Radiological implications of granite of northern Pakistan, J. Radiol. Prot.: 28, 387-399 (2008).

Baeza, A., M. del Río, C. Miró y J. Paniagua; Natural radionuclide distribution in soils of Cáceres (Spain): dosimetry implications, J. Environ. Radioactiv.: 23, 19-37 (1994).

Beretka, J. y P.J. Mathew; Natural radioactivity in Australian building materials, industrial waste and by-products, Health Phys.: 48, 87-95 (1985).

Chong, C.S. y G.U. Ahmad; Gamma activity in building materials in West Malaysia. Health Phys.: 43, 272-273 (1982).

Evans, R.D.; Engineer's guide to elementary behaviour of radon daughters. Health Phys.: 17, 229252 (1969)

Eisenbud, M.; Environmental radioactivity from natural and military sources , $3^{\mathrm{a}}$ edición, 152 , Academic Press, New York, USA (1987).

Espinosa, G., J.L. Golzarri, I. Gamboa y I. Jacobson; Natural radioactivity in Mexican building materials by SSNTD, Nucl. Tracks Radiat. Meas.: 12, 767-770. (1986).

EC-EUROPEAN COMMISSION, Radiation Protection 112-radiological protection principles concerning the natural radioactivity of building materials. Directorate-General Environment, Nuclear Safety and Civil Protection (1999)

Farai, I.P. y J.A. Ademola; Radium equivalent activity concentrations in concrete building blocks in eight cities in Soutwestern Nigeria. J. Environ. Radioactiv.: 79, 119-125 (2005).

Hewamanna, R., y otros cuatro autores; Natural radioactivity and gamma dose from Sri Lankan clay bricks used in building construction, Appl. Radiat. Isot.: 54, 365-369 (2001).

ICRP, Internacional Commision on Radiological Protection. Recommendations of ICRP, Publication 26, Pergamon Press, Oxford, UK (1977)

INTROMAC, Instituto Tecnológico de Rocas Ornamentales y Materiales de Construcción (en línea). http://www.intromac.com/ (2009).

Khan, K., M. Aslam, S.D. Orfi y H.M. Khan; Norm associated radiation hazards in bricks fabricated in various localities of the North-West Frontier Province (Pakistan). J. Environ. Radioactiv.: 58, 59-66 (2002)

Kathren, R.L.; NORM sources and their origins. Appl. Radiat. Isotopes: 49, 149-168 (1998). 
Krieger, R. ; Radioactivity of construction materials. Betonwerk Fertigteil-Tech. 47, 468-473 (1981).

Kumar, A., M. Kumar, B. Singh y S. Singh; Natural activities of ${ }^{238} \mathrm{U},{ }^{232} \mathrm{Th}$ and ${ }^{40} \mathrm{~K}$ in some Indian building materials, Radiat. Meas.: 36, 465-469 (2003)

Lee, E. M., G. Menezes y E.C. Finch; Natural radioactivity in building materials in the Republic of Ireland, Health Phys.: 86, 378-383 (2004).

Malanca, A., V. Pessina y G. Dallara; Radionuclide content of building materials and gamma ray dose in dwellings of Rio-Grande Do-Norte, Brazil, Radiat. Protect. Dosim.: 48, 199-201 (1993).

Mollah, A.S., G.U. Ahmad, S.R. Hussain y M.M. Rahman; The natural radioactivity of some building materials used in Bangladesh, Health Phys.: 50, 849-851 (1986).

Mustonen R. ; Natural radioactivity in, and radon exhalation from, Finnish building materials, Health Phys.: 46, 1195-1203 (1984).

Niewiadomski T. y M. Olszeqska; Population exposures due to current building technology in Krawkow, Poland, Radiat. Protect. Dosim.: 12, 293-296 (1985).

Oresegun, M.O. y A.I. Babalola; Annual indoor dose burden estimates in dwellings built in Nigeria with radioactive $U$-Th rich tailings, Actas de la Conferencia Internacional sobre Protección de la Radiación en Energía Nuclear, 2, 159-166, Viena-Austria, 18 al 22 Abril (1988)

Papastefanou C., M. Manolopouloy y S. Charalambou; Exposure from the radioactivity in building materials, Health Phys.: 47,775-783 (1984).

Proffijin A., R. Bourgoignie, R. Marijns y R. Jacobs; Laboratory measurement of radon exhalation and diffusion, Radiat. Protect. Dosim.: 7, 77-79 (1984).

SIGEO, Sistema de Información Geológico Minero de Extremadura. Junta de Extremadura (en línea). http://sinet3.juntaex.es/sigeo/web/asp/sgmapsearch.asp?id=1 (2009)

Sciocchetti, y otros cuatro autores; Indoor measurement of airbone natural radioactivity in Italy, Radiat. Protect. Dosim.: 7, 347-351 (1984).

Schmus, W.R.V.; Natural radioactivity of crust and mantle, Global Earth Physics: A handbook of Physical Constants, ed. T.J. Ahrens (Washington, DC: American Geophysical Union) (1995)

Sorantin, H. y F. Steger; Natural radioactivity of building materials in Austria, Radiat. Protect. Dosim.: 7, 59-61 (1984).

Tufail, M., Nasim-Akhatar, A. Sabiha-Javied y Tehsin-Hamid; Natural radioactivity hazards of building bricks fabricated from saline soil of two districts of Pakistan, J. Radiol. Prot.: 27, 481-492 (2007).

UNSCEAR (United Nations Scientific Committee on the Effects of Atomic Radiation), Sources and effects of ionizing radiation. Volumen I: Sources Report to General Assembly, with Scientific Annexes. United Nations, New York, USA (2000)

Xinwei L.; Natural radioactivity in some building materials of Xi'an, China, Radiat. Meas.: 40, 94-97 (2005).

Zikovsky L. y G. Kennedy; Radioactivity of building materials available in Canada. Health Phys.: 63 , 499-502 (1992). 